\title{
Solubility and dissolution rate enhancement of ibuprofen by co-milling with polymeric excipients
}

Amjad Hussain, Geoff Smith*, Karrar A. Khan, Nadeem Irfan Bukhari, Nicholas I. Pedge, Irina Ermolina

\begin{tabular}{|l|l|}
\hline Journal & $\begin{array}{l}\text { European Journal of Pharmaceutical } \\
\text { Sciences }\end{array}$ \\
\hline Date Received & 15 March, 2018 \\
\hline Date Revised & 15 June, 2018 \\
\hline Date Accepted on & 01 August, 2018 \\
\hline
\end{tabular}

Cited as:

Hussain, A, Smith, G., Khan K. A., Bukhari, N. I., Pedge N.I.,and Ermolina, I. (2018). Solubility and dissolution rate enhancement of ibuprofen by co-milling with polymeric materials. European Journal of pharmaceutical Sciences 123, 395-403.

${ }^{*}$ Corresponding author:

Prof. Geoff Smith

Leicester School of Pharmacy, De Montfort University, Leicester, LE1 9BH

gsmith02@dmu.ac.uk 


\section{Abstract}

The aim of this study was to enhance the kinetic solubility and dissolution rate of ibuprofen by co-milling with different excipients and to establish the underlying mechanism(s) for such enhancement. In the first-part, two excipients (HPMC and soluplus) were selected from seven, and the optimal ball-milling parameters of speed and time $(18 \mathrm{~Hz}, 15 \mathrm{~min}$ ) determined based on solubility-enhancement and flow-ability criteria. In the second-part, co-milling of different weight-ratios of ibuprofen-to-excipient was carried out and solubility and dissolution rates determined. Mechanisms of biopharmaceutical enhancement were studied by SEM, laser diffraction, DSC, and FTIR analysis of the co-mixtures. Ibuprofen solubility $(0.09 \mathrm{mg} / \mathrm{mL}$ for unmilled) was increased by factors of 4-5 and 10-20 for HPMC and soluplus, respectively. The weakening of crystals, stabilization of the amorphous phase and an increase in solid-state hydrogen bonding are the likely mechanisms for this enhancement. Reductions in Q70\% dissolution time were also observed, by a factor of four and seven for ibuprofen:HMPC and ibuprofen:soluplus co-milled mixtures, respectively. In both, cases there were similar reductions in particle size, dispersibility and degree of amorphization and so the enhanced dissolution rate for soluplus, over that for HPMC, must be due to the additional solubilisation contribution to the kinetic solubility provided by soluplus.

Key words: Poor soluble drugs, ball milling, solubility enhancement, dissolution rate, particle size, amorphous content.

\section{Abbreviations $^{1}$}

${ }^{1}$, Physical mixture (PM), milled physical mixture (mPM), Absorbance (A) 


\section{Introduction}

Milling and co-milling (which is defined as milling in the presence of an excipient) are well known techniques that have a positive influence on the kinetic solubility and dissolution rate of sparingly soluble drugs (Jagadish et al., 2010; Szafraniec et al., 2017). These procedures have been shown to provide a simple, efficient and economical method that does not require any particularly sophisticated equipment (Fisher, 2007). Moreover, the method has less of an environmental impact as it does not require the use an organic solvent (Friedrich et al., 2005). Co-milling combines the advantages of a reduction in particle size and the amorphization of a crystalline drug substance, which is a benefit of conventional milling of single materials, but with the additional benefits of improved wettability and solubilization that are provided by the co-milled excipient (Mosharraf et al., 1995). Furthermore, it may also prevent aggregation, by the surface coverage of the charged particles (produced by milling), while stabilizing the amorphous phase in the solid state and reducing the mechanical/thermal degradation of drugs by moderating the effect of the heat generated on milling (Lin et al., 2010).

Solubility is a physicochemical property of substance, which depends on the thermodynamic properties of the crystal lattice (i.e. the bonding energies which define the melting point) and the balance between solute-solute and solute-solvent (solvation) interactions in the solution state. The solute-solvent interactions may be changed by adding other chemicals to the solvent, for example surfactants which providing a micellar environment for the solubilisation of the drug. It is also important to remember that the solubility of a milled crystalline material, containing metastable (partially amorphous) phase, represents the kinetic solubility rather than thermodynamic/equilibrium solubility (Brittain, 2014).

The rate of dissolution, which defines the rate of mass transfer from the crystalline state to the dissolved (solution state) is coupled to the solubility but is also impacted by attributes of the material such as the particle size distribution (surface to volume ratio), surface tension (which influences the wettability of the surface) and the physical form (i.e. whether in a crystalline or amorphous state). In the case of an amorphous solid (which is often present in a milled material) the supersaturated solution (i.e. one which exceeds the thermodynamic equilibrium solubility) can be obtained for a short time until the system relaxes and returns to the thermodynamic equilibrium (when the excess drug precipitates from solution). The presence of an excipient in the solution or from co-milling with excipient can affect both the solubility of drug (due to the 
alteration of the liquid medium and presence of more complex interactions between solvent, excipient and drug molecules) and the dissolution rate (including the relaxation time of oversaturated solution). An extension of the oversaturated state of the solution for few hours is desirable in order to increase the bioavailability of drug. Co-milling process may have an impact on all three aspects: a reduction in drug particles size, a partial conversion to amorphous state and the alterations to the molecular interactions between drug and solvent.

The focus of this study is the model drug Ibuprofen. Ibuprofen is one of many propionic acid derivatives that provide analgesia through the inhibition of the enzyme cyclooxygenase (COX). It is widely used in the treatment of rheumatoid arthritis, osteoarthritis, ankylosing spondylitis and acute gouty arthritis (Brunton et al., 2006). Ibuprofen is practically insoluble in an aqueous or acidic medium (Saleh et al., 2008) resulting in poor bioavailability when administered as a conventional dosage form. The poor solubility of ibuprofen has been addressed using a variety of approaches. One of them is to reduce the particle size by milling (Plakkot et al., 2011), however; the milling of ibuprofen alone is difficult as it is a highly ductile material with a low melting point (Larsson et al., 2000). Nevertheless, the size reduction of ibuprofen has been achieved by co-milling with variety of excipients, i.e. aluminium hydroxide, kaolin and PVP (Mallick, Pattnaik, Swain, De, Saha, Ghoshal, et al., 2008; Mallick, Pattnaik, Swain, De, Saha, Mazumdar, et al., 2008). Other approaches to improve the solubility of ibuprofen include the preparation of solid dispersions with PEG (Hasnain et al., 2012) or poloxamer (Newa et al., 2008; Passerini et al., 2002) and the complexation with $\beta$-cyclodextrin (Chowdary et al., 2012; Salústio et al., 2011). In the majority of these studies, size reduction or the dispersion of the drug in an amorphous matrix were the underlying mechanisms for solubility and dissolution rate enhancement. Among the excipients used in the co-milling of ibuprofen in aforementioned mentioned studies, none would be expected to solubilise the drug.

In the recent works using soluplus for solubility enhancement, by the way of hot melt extrusion (Albadarin et al., 2017), electro-spinning (Nagy et al., 2012), micellization (Ke et al., 2017), spray drying (Herbrink et al., 2017) and freeze drying (Nagy et al., 2012), it was suggested that this excipient may be used to advantage when co-milled with drugs, as previously used to form amorphous solid dispersion (Caron et al., 2013). In addition, there are many other prospective candidate excipients, which have not been investigated to date for their potential to enhance the solubility and dissolution rate of ibuprofen. Examples of such excipients include HPMC, MCC, PVP 
and lactose, which are the widely used materials owing to their hydrophilicity and wetting properties for many other drugs (Garg et al., 2009; Vogt, Kunath, et al., 2008; Vogt, Vertzoni, et al., 2008).

The objectives of this study are the enhancement of solubility and dissolution rate of ibuprofen and to understand the mechanisms involved. The development of method for assaying ibuprofen in the presence of interfering substance was also an objective of this study.

There are two phases to this work; In the first (screening) phase, in which a sub-set of excipient type and processing conditions (including the speed and time of milling) were selected on the basis of best outcomes in terms of processing properties (like flowability) and solubility of the drug. In the second (extended) phase, the optimal process parameters of speed and time were applied to prepare co-milled binary mixtures of ibuprofen with the sub-set of excipient and the effect on solubility and dissolution rate of ibuprofen determined. These co-milled mixtures were then characterized by various analytical techniques in order to establish the mechanism of solubility and dissolution rate enhancement.

\section{Materials and methods}

Ibuprofen was purchased from Fischer chemical, UK. Soluplus (a graft co-polymer of PEG) and lutrol F-68 (a block co-polymer non-ionic surfactant consisting of Poly-oxyethylene-(POE-) and Polyoxypropylene-(POP-) units) were obtained from BASF, UK. Polyvinyl pyrrolidone (PVP K30) (Jiaozou Fine Chemical, China), hydroxy-propylmethyl cellulose (HPMC-E5) (Ashland, US), microcrystalline cellulose (MCC), PEG-6000, lactose monohydrate were obtained from Merck, Germany. All excipients were of pharmaceutical grade and were used as received from the suppliers.

\subsection{Assay of ibuprofen in co-milled mixtures}

A $0.05 \% \mathrm{w} / \mathrm{v}$ solution of the un-milled ibuprofen in phosphate buffer of $\mathrm{pH} 7.4$ was prepared and its UV spectrum was measured between 200-400 nm in UV spectrophotometer (2550, Schimadzou, Japan). From this spectrum, the wavelength of maximum absorbance $\left(\lambda_{\max }\right)$ was selected based on the highest clear peak (see Section 3.1).

In order to determine any interference in UV absorbance of ibuprofen by co-milled excipients, the UV spectrum of each excipient $(0.05 \% \mathrm{w} / \mathrm{v})$ was also measured and overlaid on the spectrum 
of ibuprofen (see Fig. 1). The interference as detected by soluplus was corrected by applying two wavelength assay approach or multivariate least square approach (see Section 3.3).

\subsection{Screening phase}

\subsubsection{Co-milling of ibuprofen with excipients}

At the initial stage of this study, an optimum milling speed and time was determined and a subset of these excipients selected based on the binary mixtures that provided highest solubility while maintaining optimal flowability (Section 3.5).

For this purpose soluplus was used as trial excipient (as it was the only excipient that has shown the prominent effect on solubility of drug on changing the concentration while maintaining the flowability) and it was co-milled with ibuprofen in an oscillatory ball mill (MM 301, Retsch, Germany), according to following protocol.

i) For the selection of milling speed, Ibuprofen and soluplus (1:0.5 ratio) were co-milled at three different speeds, viz. 15, 18 and $25 \mathrm{~Hz}$, for $15 \mathrm{~min}$. The milling time of $15 \mathrm{~min}$ was selected on hit and trail basis to avoid melting of this drug.

ii) For the selection of milling time, the mill speed was fixed (i.e. $18 \mathrm{~Hz}$, see results in Section 3.5) and ibuprofen was co-milled with soluplus (1:0.5 ratio) for 5, 10, 15 and $30 \mathrm{~min}$.

iii) For the selection of best excipient for co-milling, Ibuprofen was co-milled with different excipients (soluplus, HPMC, lutrol, PVP, MCC, lactose and PEG-6000) with 1:1 ratio at the selected speed and time (18 Hz and $15 \mathrm{~min}$, see results Section 3.1).

The solubility of co-milled mixtures was determined as per method described in Section 2.2.2.

\subsubsection{Solubility studies}

The well-known shake the flask method (Nandi et al., 2003) was used to determine the solubility of ibuprofen in distilled water ( $\mathrm{pH}$ 6.1). For this purpose, an excess quantity of ibuprofen or its mixtures equivalent to $200 \mathrm{mg}$ ibuprofen was added to a $100 \mathrm{~mL}$ conical flask containing $50 \mathrm{~mL}$ of the distilled water. The flasks were capped and shaken at $100 \mathrm{rpm}$ on a multi-flask shaker (Heidolph Unimax 2010, Germany) at $\sim 25{ }^{\circ} \mathrm{C}$ temperature. The samples were collected after 24 $\mathrm{hr}$, filtered through $0.45 \mu \mathrm{m}$ syringe filters (Millipore, US) and diluted appropriately and the concentration of ibuprofen was determined by UV spectrophotometry. 
Standard solutions of known concentrations of ibuprofen in distilled water were also prepared and used to construct calibration models. The calibration standard solutions, designed in adherence to Beer-Lambert law (i.e. $A<1$ ) were linear in the range of 0.005 to $0.08 \mathrm{mg} / \mathrm{mL}$ in distilled water, with value of correlation coefficient near unity and value of intercept on the ordinate, near to zero.

\subsection{Extended phase}

In this phase, the selected excipients (i.e. soluplus and HPMC) and process parameters (milling speed $18 \mathrm{~Hz}$ and time $15 \mathrm{~min}$ ) were used to produce co-milled mixtures with ibuprofen. The solubility and dissolution rates of these mixtures were determined and these were evaluated with different analytical techniques to establish the possible mechanism for solubility and/dissolution rate improvement (if any).

\subsubsection{Co-milling of ibuprofen with selected excipients}

Ibuprofen was co-milled with soluplus and HPMC in different drug to excipient ratios (i.e. 1:0.25, 1:0.5, 1:0.75 and 1:1) in order to determine the effect of excipient concentration on the solubility and dissolution rate of drug.

The physical mixtures of ibuprofen with these excipients and the physical mixtures of the premilled materials (abbreviated as PM and PMPM, respectively) were also prepared for the purpose of a comparison with co-milled mixtures. The PM was prepared by simple mixing of both components with spatula in a weighing boat (Barzegar-Jalali et al., 2010) and the PMPMs were prepared by milling the ibuprofen and excipients separately in a ball mill then mixing these in 1:1 ratio.

\subsubsection{Solubility studies}

Method already described under Section 2.2.2.

\subsubsection{Dissolution rate Studies}

Ibuprofen and its co-milled mixture with different excipients, equivalent to $200 \mathrm{mg}$ ibuprofen, were filled in colourless hard gelatine capsule shells and subjected to dissolution studies in a USP type I (paddle) apparatus (DT-700, Erweka Germany). $900 \mathrm{~mL}$ of phosphate buffer (pH 7.4) was selected as the dissolution medium and the temperature maintained at $37 \pm 0.4^{\circ} \mathrm{C}$. The paddle 
speed was set up at $50 \mathrm{rpm}$. Aliquots of $5 \mathrm{~mL}$ were withdrawn at intervals of $5,10,20,30,45,60$ and $90 \mathrm{~min}$ and replaced with the equal volume of the fresh dissolution medium in order to maintain a constant volume. Each sample was filtered through a $0.45 \mu \mathrm{m}$ syringe filter and then diluted adequately (i.e. $A_{\max }<1$ ) for assay by UV spectrophotometry.

\subsubsection{Particle size determination}

The particle size distribution (PSD) of un-milled ibuprofen and its co-milled mixtures with HPMC or soluplus (1:1) was determined with dry dispersion laser diffraction technique according to the method described in literature. (Krause et al., 2011). The powder was dispersed in compressed air at a pressure of $3 \pm 0.05$ bar using a dispersion unit, RODOS (Sympatec, Germany), dispensed at the feed rate of $60 \mathrm{~mm} \mathrm{sec}^{-1}$ using a micro-dosing unit, ASPIRO (Sympatec, Germany) and measured under the pressure of $\sim 0.5$ bar with a laser diffractometer (HELOS H1360, Sympatec, $\mathrm{GmbH}$, Germany) fitted with R5 lens (Sympatec) that can measure the particle size between 4.5 to $875 \mu \mathrm{m}$. Measurements were taken in triplicate and results presented as average values.

\subsubsection{Scanning electron microscopy (SEM)}

SEM images of un-milled, milled and co-milled Ibuprofen samples were obtained on a ZEISS EVO HD 15 scanning electron microscope (Carl Zeiss, NTS Ltd. Cambridge, UK) according to the method described (Qiao et al., 2013). The samples were mounted on the carbon adhesive tape fixed on aluminium stubs (Agar Scientific Ltd., Stansted, UK) and flushed with air. The SEM images were taken at the electron beam voltage of $10 \mathrm{KV}$.

\subsubsection{Differential scanning calorimetry (DSC)}

DSC experiments of un-milled ibuprofen and its co-milled mixtures with different excipients were performed according to the method described in our previous study (Smith et al., 2015). The sample was analysed over the temperature range from 25 to $150^{\circ} \mathrm{C}$ at a ramp rate of $20^{\circ} \mathrm{C} / \mathrm{min}$. In order to compare the results of milled ibuprofen with that of a $100 \%$ amorphous sample, a sample of crystalline ibuprofen contained in non-hermetically sealed DSC pan was vitrified by heating in an oven at $90{ }^{\circ} \mathrm{C}$ for $10 \mathrm{~min}$ then quenched by dipping it in the liquid nitrogen. This pan was loaded in a pre-cooled DSC furnace and heated from $-60^{\circ} \mathrm{C}$ to $110^{\circ} \mathrm{C}$. 


\subsubsection{Attenuated total reflectance (ATR) spectroscopy}

The IR spectra of un-milled, milled and co-milled ibuprofen were recorded in Bruker Alpha -FTIR Spectrophotometer (Bruker, Japan) fitted with a Smart Performer, platinum ATR accessory. The data were analyzed by Alpha Opus Software. The instrument was configured with an ATR sample cell containing a diamond crystal with scanning depth of $2 \mu \mathrm{m}$. Sample powder was placed on the surface of crystal and secured in place with clutch type lever. Each sample was scanned for 20 times against air between $4000-400 \mathrm{~cm}^{-1}$ at the resolution of $2 \mathrm{~cm}^{-1}$. 


\section{Results and discussion}

\subsection{UV spectra of ibuprofen and excipients}

A $0.05 \% \mathrm{w} / \mathrm{v}$ solution of the un-milled and milled ibuprofen in $\mathrm{pH} 7.4$ phosphate buffer showed a well-defined, large peak at $221 \mathrm{~nm}$ (visible in the diluted samples - not shown here) and two other peaks; one at $264 \mathrm{~nm}$ and a second at $273 \mathrm{~nm}$ along with a shoulder at $258 \mathrm{~nm}$ (Appendix 1). The overlaid spectra of $0.05 \% \mathrm{w} / \mathrm{v}$ ibuprofen and excipients in phosphate buffer have shown that, lutrol, MCC, PEG-6000, Lactose and HPMC solutions have negligible absorbance over the entire UV range. In contrast, PVP and soluplus solutions exhibit a high absorbance, with the former only at wavelengths below $230 \mathrm{~nm}$ while the later over the entire range of wavelengths $(210-330 \mathrm{~nm})$.

\subsection{Selection of UV absorbance peak for the ibuprofen assay}

The absorbance peak of ibuprofen at $221 \mathrm{~nm}$ has been reported previously in many studies (Nokhodchi et al., 2010). However, the solutions of soluplus and PVP exhibit absorbance values greater than $0.7(A>0.7)$ below $230 \mathrm{~nm}$. This precluded the use of the absorbance peak of ibuprofen at $221 \mathrm{~nm}$ in the development of the UV-assay of ibuprofen in the presence of these two excipients. For consistency the peak at $221 \mathrm{~nm}$ was also not used for the other excipients. In order to select the analytical wavelength, the absorbance of ibuprofen was determined at three potential analytical wavelengths 258,264 and $272.4 \mathrm{~nm}$ (Table 1). The results indicated that lutrol, HPMC and PVP have only 1-2\% relative absorbance at these three wavelengths for ibuprofen. Therefore, the wavelength of maximum absorbance $(\lambda \max )$ i.e. $264 \mathrm{~nm}$, was selected for the assay of ibuprofen in the presence of these excipients.

On the other hand, the UV spectrum of soluplus solution has shown a significant absorbance over the entire wavelength range, which increases towards lower wavelengths (Appendix 1). This spectrum, when overlaid on the spectrum of same concentration of ibuprofen shows higher absorbance than ibuprofen solution towards higher wavelength (> $285 \mathrm{~nm}$ ). The absorbance of ibuprofen starts increasing and becomes higher than the soluplus solution absorbance towards lower wavelengths. Therefore, two wavelengths are selected for the estimation of ibuprofen in the presence of soluplus: first, $264 \mathrm{~nm}$ which is the $\lambda_{\max }$ of ibuprofen and has the lowest relative 
absorbance of soluplus among its three potential wavelengths (Table 1); second, $287 \mathrm{~nm}$ at which soluplus shows higher absorbance.

Table 1: a) Relative absorbance of the excipients as compared to the absorbance of ibuprofen at three potential wavelengths of ibuprofen

\begin{tabular}{cccc}
\hline & \multicolumn{3}{c}{ Relative Absorbance (\%) } \\
& $258 \mathrm{~nm}$ & $264 \mathrm{~nm}$ & $272.4 \mathrm{~nm}$ \\
\hline HPMC & 1.4 & 1.1 & 1.2 \\
\hline lutrol & 0.4 & 0.3 & 0.4 \\
MCC & 1.1 & 0.9 & 0.7 \\
Lactose & 0.2 & 0.4 & 0.4 \\
\hline PVP & 2.1 & 1.5 & 1.5 \\
PEG-6000 & 0.4 & 0.3 & 0.4 \\
\hline Soluplus & 55.2 & 40.5 & 45.2 \\
\hline
\end{tabular}

\subsection{Correction of the interference (UV absorbance) of soluplus in the quantitative} determination of ibuprofen in the mixture

\subsubsection{Two wavelength assay}

The two wavelength assay approach, which is described in literature as absorbance ratio method (Erk, 2000) or simultaneous equation method (Nallasivan et al., 2010; Patil et al., 2009) was applied with minor modifications and generally involves the measurement of the absorbance of both the drug and interfering excipient (i.e. ibuprofen and soluplus) at two different wavelengths; $\lambda 1$ and $\lambda 2$. The calibration curves are constructed by plotting these absorbance values against concentrations of both single component solutions of ibuprofen drug $x(d)$ and soluplus excipient $x(e)$ respectively. From this calibration curve, the absorption of both drug and excipient at two wavelengths can be described by the following relations:

At $\lambda 1$

$A(d, \lambda 1)=\mathbf{m}(\mathbf{d}, \lambda \mathbf{1}) \cdot \mathbf{x}(\mathbf{d})$

$\mathbf{A}(\mathbf{e}, \lambda \mathbf{1})=\mathbf{m}(\mathbf{e}, \boldsymbol{\lambda} \mathbf{1}) \cdot \mathbf{x}(\mathbf{e})$

At $\lambda 2$ 
$A(d, \lambda 2)=m(d, \lambda 2) \cdot x(d)$

$A(e, \lambda 2)=m(e, \lambda 2) \cdot x(e)$

Where $m(d, \lambda 1), m(d, \lambda 2)$ are the gradient of curve drawn for the drug at $\lambda 1$ and $\lambda 2$; while, $m(e$, $\lambda 1), m(e, \lambda 2)$ are the slope of excipient at $\lambda 1$ and $\lambda 2$ respectively. The total absorbance (T-total) of the mixture of drug and excipient at both wavelengths is measured as $A(T, \lambda 1)$ and $A(T, \lambda 2)$ respectively. The individual contributions to the total absorbance at $\lambda 1$ and $\lambda 2$ are presented as:

$\mathbf{A}(\mathbf{T}, \lambda \mathbf{1})=\mathbf{A}(\mathbf{d}, \boldsymbol{\lambda 1})+\mathbf{A}(\mathbf{e}, \lambda \mathbf{1})$

$A(T, \lambda 2)=A(d, \lambda 2)+A(e, \lambda 2)$

Now by substitution of the expressions for the individual absorbance of both components from equations 1 to 4 in equation 5 and 6 , gives the following expression for total absorption as:

$A(T, \lambda \mathbf{1})=\mathbf{m}(\mathbf{d}, \lambda \mathbf{1}) \cdot \mathbf{x}(\mathbf{d})+\mathbf{m}(\mathbf{e}, \lambda \mathbf{1}) \cdot \mathbf{x}(\mathbf{e})$

$A(T, \lambda 2)=\mathbf{m}(\mathbf{e}, \lambda 2) \cdot \mathbf{x}(\mathbf{e})+\mathbf{m}(\mathbf{e}, \lambda 2) \cdot \mathbf{x}(\mathbf{e})$

The unknown concentration of drug $x(d)$ in the mixture is determined by applying the following equation obtained from re-arranging and substituting the equations 7-8:

$\mathbf{x}(\mathbf{d})=\left[\frac{\mathrm{A}(\mathrm{T}, \lambda 2) \cdot \mathbf{m}(\mathbf{e}, \lambda 1)-\mathrm{A}(\mathrm{T}, \lambda \mathbf{1}) \cdot \mathbf{m}(\mathbf{e}, \lambda 2)}{\mathbf{m}(\mathbf{d}, \lambda 2) \cdot \mathbf{m}(\mathbf{e}, \lambda \mathbf{1})-\mathbf{m}(\mathbf{e}, \lambda 2) \cdot \mathbf{m}(\mathbf{d}, \lambda \mathbf{1})}\right]$

\subsubsection{Multivariate Least Square Approach}

The equation 9 works appropriately for non-interacting materials. However, while assaying the ibuprofen in the presence of soluplus, it was found that the UV spectrum of 1 to 1 mixture of ibuprofen and soluplus (1:0.5) solutions was higher than the theoretical spectrum, i.e. mathematical sum of individual spectra of both materials (Appendix 2). This might be due to light scattering effect of soluplus that has formed micelles in the solution. Therefore, the interference by the soluplus could not be corrected simply by two wavelength method.

Another approach, i.e. multivariate analysis, was applied to examine the spectrum of such mixture. The least-squares solution to calculate the contribution (concentration) of each component from the mixture spectrum, using reference spectra may be given by the Equation 10. 


$$
c=\left(S^{\prime} S\right)^{-1} S^{\prime} \mathbf{y}
$$

Equation 10

where $\mathrm{c}$ is the concentration factor, which represents the exact contribution of the individual components in the mixture of ibuprofen and soluplus and $\mathrm{y}$ is the measured spectrum of the mixture of both components. An advantage of the multivariate approach is that the component concentrations are estimated using many spectral variables and thus helps to average out the measurement noise present in each variable.

The least squares approach can be applied using the original spectra; or first derivative spectra (calculated using the Savitzky-Golay method, using a 15-point window and a second-order polynomial). The spectra of both ibuprofen and soluplus with concentration of $0.25 \mathrm{mg} / \mathrm{mL}$ and $0.125 \mathrm{mg} / \mathrm{mL}$, respectively (1:0.5 ratio) were used to create a two column matrix $\mathrm{S}$. In case of first derivative spectra, the matrix $\mathbf{S}$ is constructed from first derivative reference spectra (Appendix 3a) that minimizes the spectral contribution of soluplus, and the mixture spectrum (denoted y) is also transformed to the first derivative prior to applying equation 10.

The new spectrum (derivative) predicted from the matrix based on this concentration factor overlaid the measured spectrum (Appendix 3b). The residual spectrum in this plot illustrates that the soluplus spectrum is smooth and featureless, so most of the values in the soluplus first derivative spectrum are zero. This serves to enhance the selectivity of the ibuprofen spectrum and thus provides more robust estimates of the concentration of ibuprofen. Therefore, the estimated concentrations, c can then be used to reconstruct a mixture spectrum.and was used to calculate the concentration of ibuprofen in the mixture with soluplus. The values of $\mathrm{c}$ obtained for mixture of ibuprofen and soluplus in four different ratios have been summarized in Table 2.

Table 2: Values of concentration factor and predicted concentrations calculated for ibuprofen in various mixtures with soluplus applying multivariate analysis.

\begin{tabular}{ccc}
\hline $\begin{array}{c}\text { Ibuprofen to soluplus } \\
\text { ratio }\end{array}$ & $\begin{array}{c}\text { Concentration factors } \\
\text { of ibuprofen (from } \\
\text { predicted derivative } \\
\text { (Ibu conc. } \mathbf{0 . 2 5} \mathrm{mg} / \mathrm{mL} \text { ) }\end{array}$ & $\begin{array}{c}\text { Predicted concentration of } \\
\text { lbuprofen in mixture }\left(\mathbf{c}^{*} \mathbf{0 . 2 5}\right. \\
\mathrm{mg} / \mathrm{mL})\end{array}$ \\
\hline $\mathbf{1 : 0 . 2 5}$ & 0.99 & 0.2475 \\
$\mathbf{1 : 0 . 5}$ & 0.98 & 0.2450 \\
$\mathbf{1 : 0 . 7 5}$ & 0.97 & 0.2425 \\
$\mathbf{1 : 1}$ & 0.97 & 0.2425 \\
\hline
\end{tabular}

The solubilities of ibuprofen in all other excipient-drug combinations were determined by simply taking the absorbance at the analytical wavelength of $264 \mathrm{~nm}$ and equating that to ibuprofen via the calibration curve for pure ibuprofen in distilled water. 


\subsection{Solubility of un-milled and milled ibuprofen}

The solubility of un-milled ibuprofen $(n=3)$ in distilled water at room temperature $\left(\sim 25^{\circ} \mathrm{C}\right)$ was $\sim 0.09 \mathrm{mg} / \mathrm{mL}$. Unsurprisingly, milled Ibuprofen was found to have almost the same solubility. These values were comparable or slightly higher than the reported solubility values of Ibuprofen from the literature (viz. $0.056 \pm 0.004$, (Kocbek et al., 2006) $0.09 \mathrm{mg} / \mathrm{mL}$ (Milhem et al., 2000) and $0.081 \mathrm{mg} / \mathrm{mL}$ (Saleh et al., 2008).

\subsection{Results of screening phase}

The solubility of ibuprofen increases with the milling speed (Table 3); however at the higher speed $(>18 \mathrm{~Hz}$ ), the co-milled mixture becomes sticky mass and difficult to remove from the jar of the mill. Therefore, the speed of $18 \mathrm{~Hz}$ was used for all subsequent milling experiments.

Table 3: Effect of various milling speeds on the solubility and physical state of co-milled mixtures of ibuprofen and soluplus (the milling time was $\mathbf{1 5} \mathrm{min}$ and the drug-to-soluplus ratio was constant).

\begin{tabular}{ccc}
\hline $\begin{array}{c}\text { Milling } \\
\text { speed (Hz) }\end{array}$ & $\begin{array}{c}\text { Solubility }(\mathrm{mg} / \mathrm{mL}) \\
\text { of ibuprofen }\end{array}$ & $\begin{array}{c}\text { Physical state } \\
\text { of Co-milled } \\
\text { mixture }\end{array}$ \\
\hline $\mathbf{1 5}$ & 1.21 & Powder \\
$\mathbf{1 8}$ & 1.26 & Powder \\
$\mathbf{2 5}$ & 1.89 & Sticky mass \\
\hline
\end{tabular}

The results indicate that the solubility increases with increasing the milling time from 5 min to 30 min (Table 4). However, on milling for the longer time periods (> $15 \mathrm{~min}$ ) the mixtures became sticky mass (presumably as a result of an increased temperature in the milling jar). This mixture has poor flowability and is difficult to extract from the mill, therefore the 15 min milling time was selected for the next part of this study.

Table 4: Effect of various milling times on the solubility and physical state of co-milled mixtures of ibuprofen and soluplus (the milling speed was $18 \mathrm{~Hz}$ and drug-to-soluplus ratio was constant).

\begin{tabular}{ccc}
\hline $\begin{array}{c}\text { Milling } \\
\text { Time } \\
(\mathbf{m i n})\end{array}$ & $\begin{array}{c}\text { Solubility }(\mathrm{mg} / \mathrm{mL}) \\
\text { of ibuprofen }\end{array}$ & $\begin{array}{c}\text { Physical state } \\
\text { of Co-milled } \\
\text { mixture }\end{array}$ \\
\hline $\mathbf{5}$ & 0.31 & Powder \\
$\mathbf{1 0}$ & 0.54 & Powder \\
$\mathbf{1 5}$ & 1.26 & Powder \\
$\mathbf{3 0}$ & 1.30 & sticky mass \\
\hline
\end{tabular}

Fig. 1 shows the comparison of solubilities of ibuprofen and its co-milled mixtures with different excipients. The results indicate that the solubility of ibuprofen in co-milled mixtures is higher than 
the un-milled drug alone, with the co-milled mixtures ibuprofen and HPMC, lutrol or soluplus (in a $1: 0.5$ ratio) resulting in solubilities of $0.53 \mathrm{mg} / \mathrm{mL}, 0.66 \mathrm{mg} / \mathrm{mL}$ and $1.26 \mathrm{mg} / \mathrm{mL}$, respectively, in comparison with of pure drug alone $(0.09 \mathrm{mg} / \mathrm{mL}$ ) (Saleh et al., 2008). In contrast, co-milling with lactose, PEG, PVP and MCC only had a moderate impact on the solubility of ibuprofen; with values of $0.13 \mathrm{mg} / \mathrm{mL}, 0.19 \mathrm{mg} / \mathrm{mL}, 0.12 \mathrm{mg} / \mathrm{mL}$, and $0.21 \mathrm{mg} / \mathrm{mL}$, respectively. The co-milled mixtures with lutrol and PEG-6000 were sticky mass and were not workable.

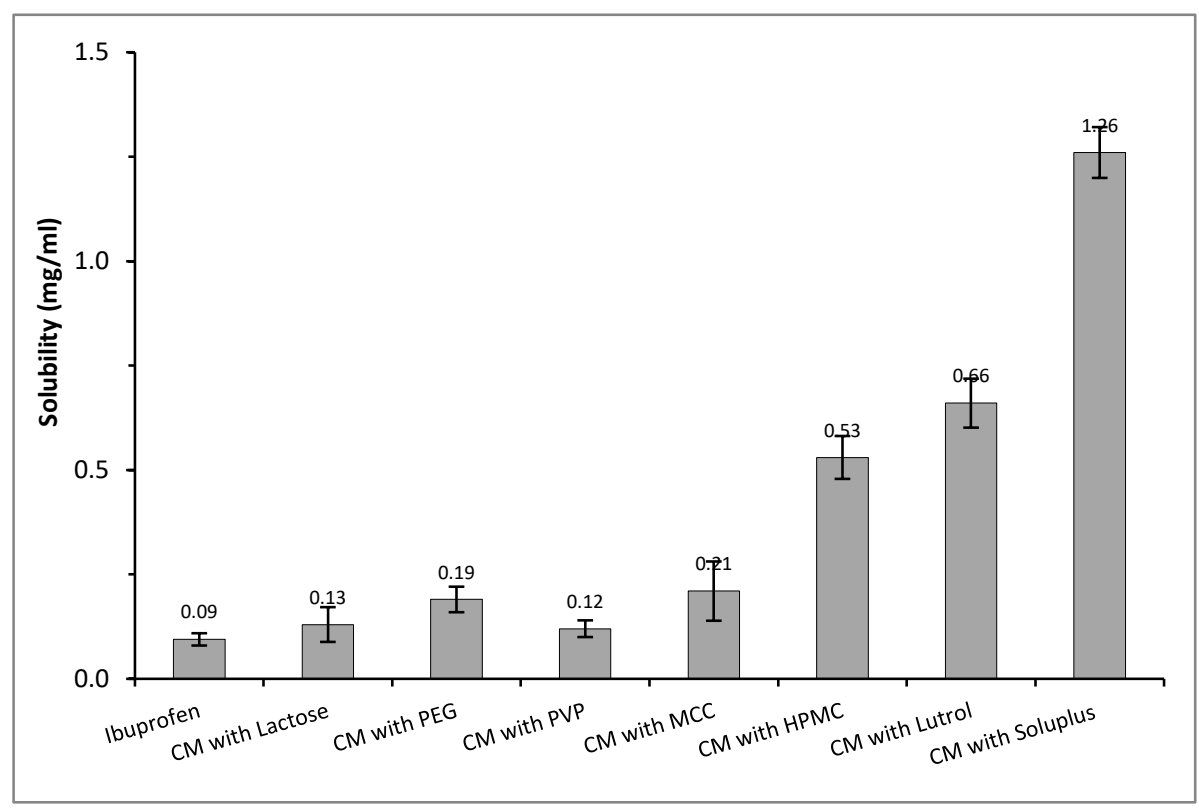

Fig. 1: Solubility values of ibuprofen (in distilled water) and its co-milled mixtures with 1:1 ratio of different excipient. The error bars represents the standard deviation from repeats of the milling processes $(n=3)$. ${ }^{*} \mathrm{CM}$ stands for co-milled mixture.

Based on the highest solubility of ibuprofen and their workable mixtures; soluplus and HMPC were selected for next phase of study.

\subsection{Results of extended phase}

\subsubsection{Effect of concentration of excipients on the solubility of ibuprofen}

The co-milled mixtures of ibuprofen and HPMC in ratios of 1:0.25, 1:0.5, 1:0.75 and 1:1, have the solubilities of $0.41,0.53,0.57$ and $0.56 \mathrm{mg} / \mathrm{mL}$, respectively (Fig. 2), while the physical mixture, even with highest ratio, i.e. 1:1 only has a solubility of $0.25 \mathrm{mg} / \mathrm{mL}$. The co-milled mixtures with different ratios of ibuprofen to Soluplus ${ }^{\circledR}$ have solubilities of $0.71,1.26,1.42$ and $1.96 \mathrm{mg} / \mathrm{mL}$ respectively and the physical mixture (1:1) $0.93 \mathrm{mg} / \mathrm{mL}$ (Fig. 5). 


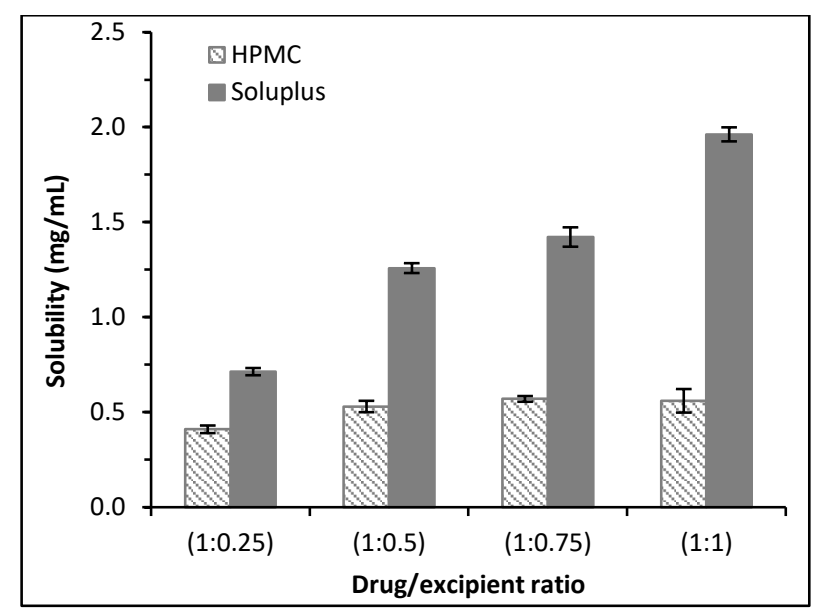

Fig. 2: Solubility values (in distilled water) of ibuprofen and its co-milled mixture with four different ratios with HPMC and Soluplus (as mentioned on $\mathrm{x}$-axis). The error bars represents the standard deviation of three values $(n=3)$.

Therefore, the solubility of ibuprofen in co-milled mixtures with HPMC is 4 to 5 times higher than the solubility of the un-milled or milled drug whereas the solubility of ibuprofen in co-milled mixtures with soluplus is 10 to 20 times higher than the solubility of the un-milled or milled drug. These trends of solubility enhancement of ibuprofen with increasing the ratios of polymers to drug are comparable to results achieved by (Madhuri Newa et al., 2008) in solid dispersion with PEG-8000. However the enhanced solubility values are $\sim 5$ times less than those obtained in dendrimer solutions (Milhem et al., 2000).

\subsubsection{Dissolution of ibuprofen and its co-milled mixtures}

The in-vitro release profile of un-milled, milled and co-milled (with Soluplus and HPMC) ibuprofen in phosphate buffer ( $\mathrm{pH} 7.4$ ) is shown in Fig. 6. The percentage of un-milled ibuprofen dissolved in first $15 \mathrm{~min}$ is $\sim 40 \%$ and $\sim 75 \%$ at $90 \mathrm{~min}$, whereas the milled (15 min) ibuprofen exhibited a slightly faster release with $\sim 45 \%$ drug dissolve in first $15 \mathrm{~min}$ and $\sim 80 \%$ in $90 \mathrm{~min}$ (Fig. 3a). This is contrary to the results already given in literature for milled ibuprofen, where a slight decrease in dissolution rate was reported (Han et al., 2011). This was probably due to the lack of agglomeration of milled particle (as discussed in SEM results) that was observed previously.

The co-milled mixtures with Soluplus exhibited the greatest release of drug i.e. 60 to $85 \%$ in first $15 \mathrm{~min}$ and $>95 \%$ after $90 \mathrm{~min}$ (Fig. 3a) which increased with the proportion of Soluplus in the mixture. The physical and milled physical mixtures of soluplus also have higher dissolution rate than the ibuprofen alone (with both the un-milled and milled drug). 
The co-milled mixtures with HPMC, in contrast, show a relatively slow release of drug that gradually increases as the amount of HPMC increases in the co-milled mixtures (Fig. 3b). The percentage release from 1:1 physical mixture was almost similar to that for the un-milled ibuprofen i.e. $\sim 40 \%$ in 15 min time. The cumulative release increases by $\sim 5 \%$ in milled physical mixture and by a further $10 \%$ in each co-milled mixture with an increase the HPMC ratio from 0.5:1 to $1: 1$ (HPMC to ibuprofen).

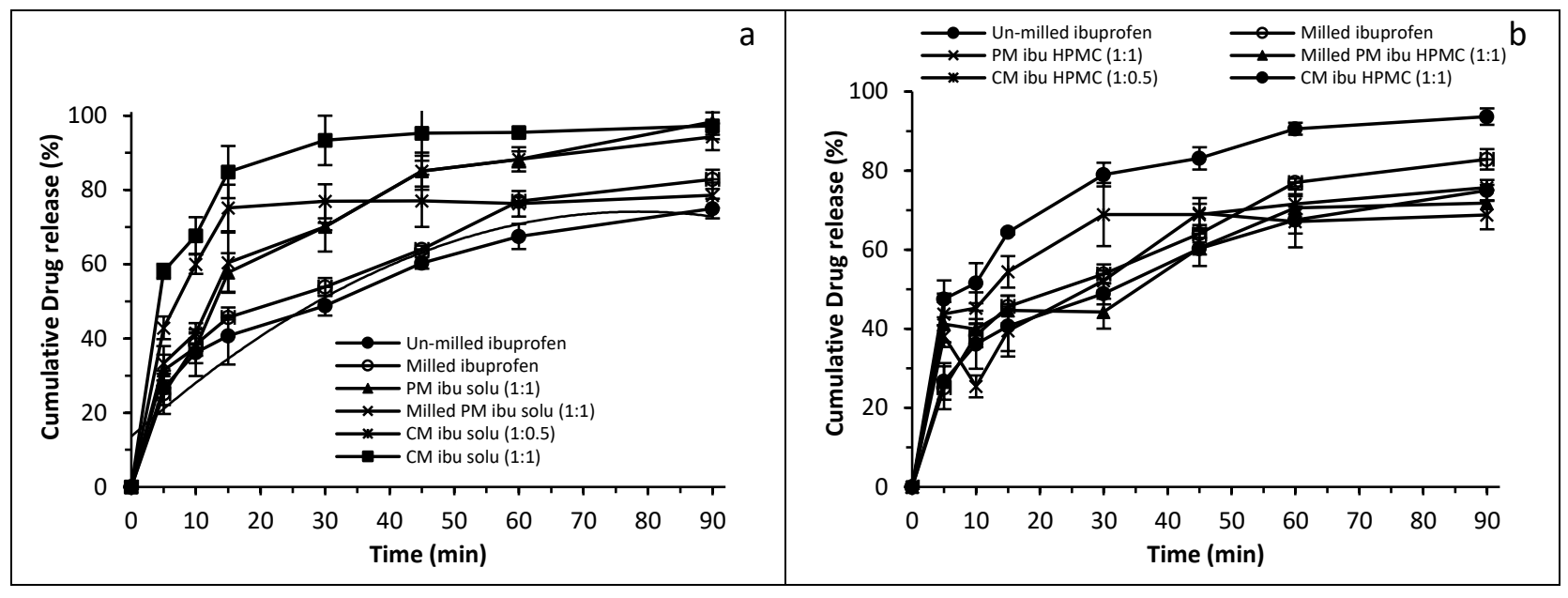

Fig. 3: Dissolution profiles (in phosphate buffer pH 7.4) of un-milled, 15min milled ibuprofen and its physical and co-milled mixtures with a) soluplus, b) HPMC. The error bars represent standard deviation with $\mathbf{n}=3$.

These results indicate that the time for $70 \%$ drug release (Q70\%) which was $\sim 72 \mathrm{~min}$ in un-milled ibuprofen, has reduced to $52 \mathrm{~min}$ in milled ibuprofen; $20 \mathrm{~min}$ in co-milled mixtures with HPMC and 10 min in co-milled mixtures with Soluplus. The dissolution rate of ibuprofen, particularly from the co-milled mixture with Soluplus, was faster than that already reported for co-milled mixture with kaolin (Mallick, Pattnaik, Swain, De, Saha, Ghoshal, et al., 2008) and aluminium hydroxide (Mallick, Pattnaik, Swain, De, Saha, Mazumdar, et al., 2008) while comparable to those co-milled with PVP (Han et al., 2011). In order to determine the mechanism of such enhancement, the co-milled mixtures were then characterized by a number of techniques.

\subsubsection{Analytics to study the changes brought by milling}

\subsubsection{Changes in particle size distribution on co-milling (Laser Diffraction Results)}

The size distribution of un-milled ibuprofen and its co-milled mixtures with HPMC and Soluplus has been presented as cumulative plots (Fig. 4). This plot indicates that in un-milled ibuprofen $\sim 50 \%$ particles have size less than $125 \mu \mathrm{m}$, while the rest of 50\% have size between 125 and 400 $\mu \mathrm{m}$. On the other hand, in samples co-milled with HPMC $\sim 80 \%$ particles have size less than 35 
$\mu \mathrm{m}$ and the co-milled mixtures with soluplus contain $\sim 90 \%$ particles of size $20 \mu \mathrm{m}$ or less. These reductions in the particle size would be accompanied by a dramatic increase in the surface area of the ibuprofen in the co-milled mixtures for both excipients and hence the observed increase in the dissolution rate of ibuprofen in the co-milled mixture.

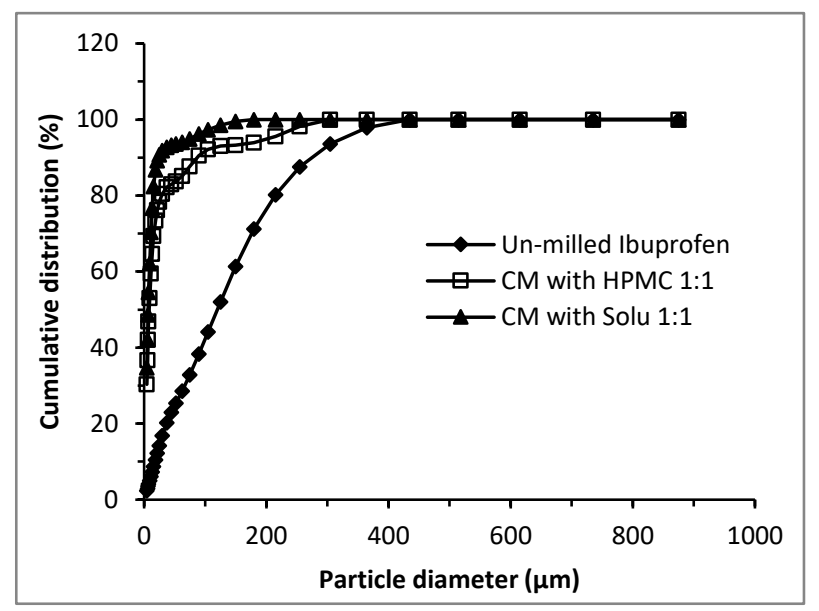

Fig. 4: Particle size plots (cumulative distribution) for un-milled Ibuprofen and its co-milled mixture with HPMC and Soluplus.

\subsubsection{Changes in size, shape and dispersibility of ibuprofen on co-milling - SEM Results}

The SEM images of ibuprofen have shown that the un-milled ibuprofen (Fig. 5a) occurs as acicular shape crystals ( 80 to $160 \mu \mathrm{m}$ in size) that are longer than width and have smooth surfaces.(Han et al., 2011). On milling ibuprofen alone, the particles are fragmented and show multiple cracks on the surface of particles (Fig. 5b), which might have provided the enlarged surface for wicking of the solvent during dissolution experiment, thereby providing additional mechanisms for the observed dissolution rate enhancement (Q70\% , 20 min reduced, see Section 3.6.2).

The co-milled mixtures of ibuprofen with soluplus and HPMC (Fig. 5c and 5d) show that the drug particles lose their more regular acicular shape while being partially embedded within what appears to be the excipient. This phenomenon might prevent the aggregation of particles, thereby enhancing dispersibility, leading to an increase in surface area exposed to the dissolution medium (Barzegar-Jalali et al., 2010) while providing additional mechanisms for wetting the drug particle surface. This might be one of the reasons for enhanced dissolution rate of drug in these co-milled mixtures. 


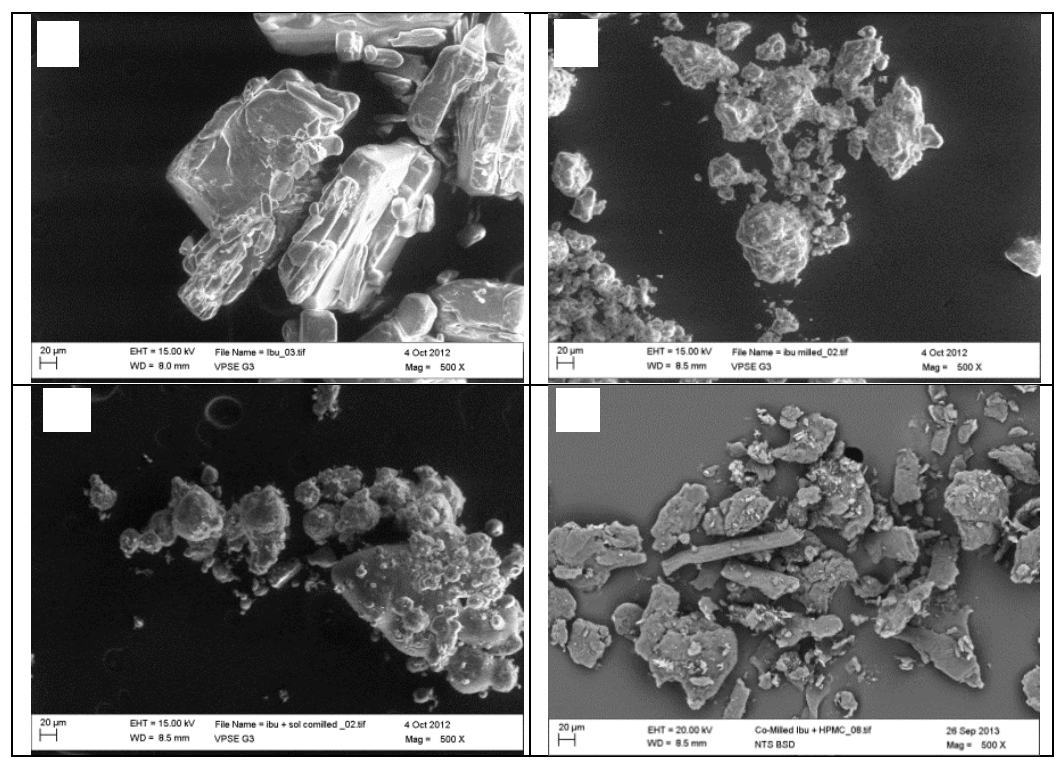

Fig. 5: SEM images of un-milled (a), 15min milled (b) ibuprofen and its co-milled mixtures with Soluplus (c) and HPMC (d) at X 500 magnification.

\subsubsection{Changes in crystallinity of ibuprofen on milling and co-Milling - DSC Results}

The DSC curve of un-milled Ibuprofen (Fig. 6a) shows a single endothermic peak at $\sim 80{ }^{\circ} \mathrm{C}$ corresponding to the melting of ibuprofen (Madhuri Newa et al., 2008). In the milled ibuprofen, the melting temperature is almost the same but the enthalpy of melting slightly decreases from $\sim 116 \mathrm{Jg}^{-1}$ to $\sim 107 \mathrm{Jg}^{-1}$ after $15 \mathrm{~min}$ of milling. The $\mathrm{T}_{\mathrm{g}}$ step, the first signature of amorphous phase, is not observed in milled ibuprofen, however the de-vitrification peak is present at almost the same temperature as observed in case of fully amorphous (quenched) ibuprofen (Fig. 6a).

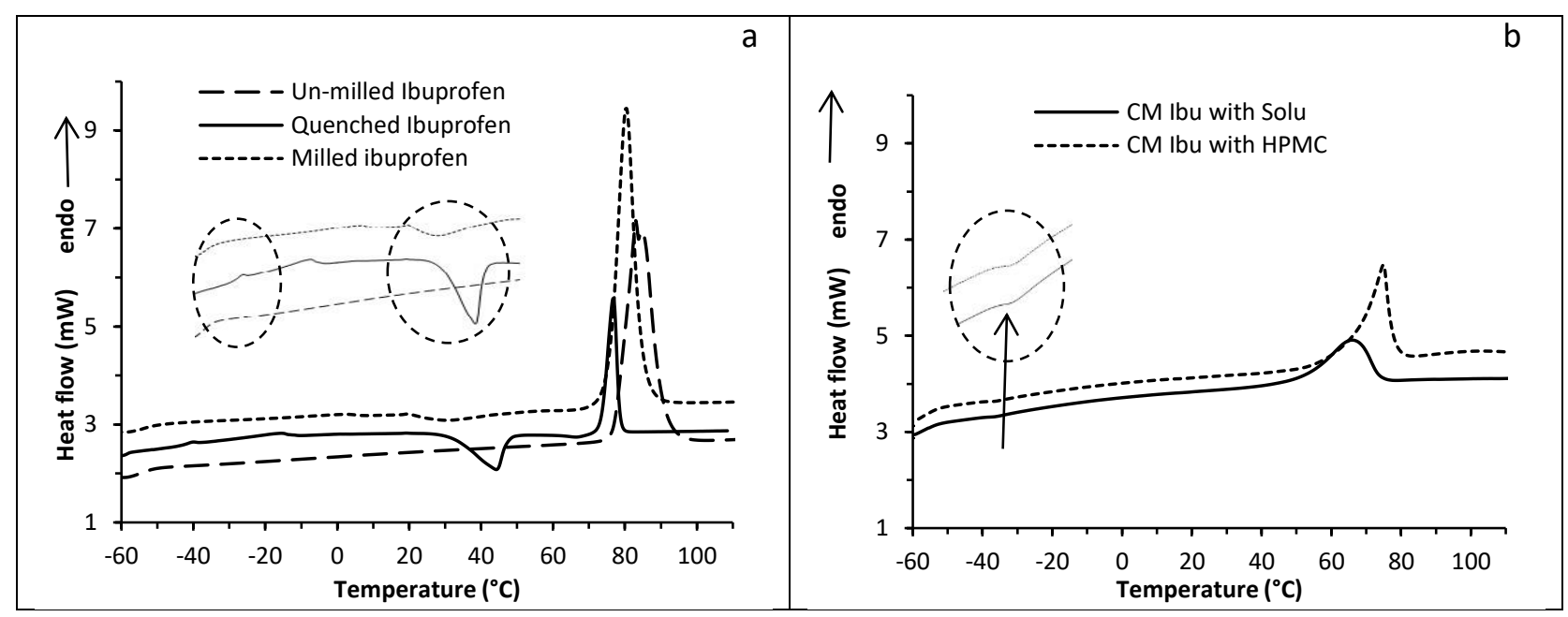

Fig. 6: DSC curves of un-milled, 15min milled and amorphous (quenched) ibuprofen (a) and that of co-milled mixtures (b), the inset shows the $\mathrm{T}_{\mathrm{g}}$ step near $-35^{\circ} \mathrm{C}$ in quenched ibuprofen and co- 
milled mixtures and de-vitrification peaks in quenched and milled ibuprofen near $25^{\circ} \mathrm{C}$ (onset) only which were otherwise absent in co-milled mixtures.

The DSC curves of co-milled mixtures of ibuprofen with HPMC and soluplus (Fig. 6b) have shown that the melting peak of ibuprofen shifts toward lower temperature and the $T_{\mathrm{g}}$ appears near -35 ${ }^{\circ} \mathrm{C}$ indicating the presence of amorphous phase (Dudognon et al., 2008). However, the devitrification peak that is present in the milled ibuprofen near $30^{\circ} \mathrm{C}$ is not observed in any of the co-milled sample. The stabilization of amorphous phase by the both co-milled excipients have also shown in literature (Pokharkar et al., 2006).

Table 5: The values of melting temperature, enthalpy of melting and \%Crystallinity for unmilled, milled ibuprofen and its co-milled mixtures with soluplus and HPMC.

\begin{tabular}{cccc}
\hline Sample & $\begin{array}{c}\text { Melting } \\
\text { peak }\left({ }^{\circ} \mathrm{C}\right)\end{array}$ & $\begin{array}{c}\text { Enthalpy of } \\
\text { melting }\left(\mathrm{Jg}^{-1}\right)\end{array}$ & $\begin{array}{c}\text { Crystallinity } \\
\text { (\%) }\end{array}$ \\
\hline Un-milled Ibuprofen & 79.7 & 116.5 & 100 \\
15 min milled & 79.7 & 108.9 & 86.7 \\
CM with HPMC 1:1 & 74.9 & 51.4 & 44.1 \\
CM with Soluplus 1:1 & 65.8 & 39.6 & 34.0 \\
\hline
\end{tabular}

The percentage residual crystallinity of ibuprofen in the co-milled mixtures is calculated from the changes in enthalpies by using the method already described in our previous studies for milled sugars (Smith et al., 2015). The results indicate that there is $55 \%$ and $65 \%$ loss in crystallinity of ibuprofen in the co-milled mixtures with HPMC and soluplus, respectively.

The shift of the melting peak of ibuprofen towards lower temperature in co-milled mixtures along with the reduction in enthalpies of melting (Table 5) has indicated that the drug crystals were weakened as previously reported by (Williams et al., 2005) for melt mixtures of ibuprofen that might be responsible for the increased solubilities and hence dissolution rates of co-milled mixtures as compared to drug alone.

\subsubsection{Molecular interaction of ibuprofen with co-milled excipients/ATR Results}

The IR spectrum of un-milled ibuprofen has shown an intense, a well-defined peak at $\sim 1704 \mathrm{~cm}^{-}$ ${ }^{1}$ corresponding to the carbonyl-stretching of propionic acid group, a characteristic band from 800 to $1500 \mathrm{~cm}^{-1}$ due to hydrogen bonding as ibuprofen forms dimer and a spectral band between 2800 and $3000 \mathrm{~cm}^{-1}$ due to the stretching of $-\mathrm{OH}$ bond (Newa et al., 2008).

The IR spectra of 1:1 co-milled mixtures of ibuprofen with HPMC and soluplus (Fig. 7a) are almost similar to that of un-milled ibuprofen, except that the spectral band near $3000 \mathrm{~cm}^{-1}$ decreases in 
size, while the stretching vibration of carbonyl peak near $1700 \mathrm{~cm}^{-1}$ was still present in co-milled mixtures indicating that the drug's crystalline form was not completely lost during co-milling (Fig. 10a). The carbonyl stretching peak that is relatively broad in the un-milled ibuprofen becomes sharper and shifts towards higher wavenumbers on milling the drug alone and co-milling it particularly with soluplus (Fig. 7b).

The shift of carbonyl peak toward higher wavenumbers in milled ibuprofen and its co-milled mixture with Soluplus suggests that there is a change in the dimer structure and hydrogen bonding (Nokhodchi et al., 2010). This might partly explain the solubilization action of soluplus, in the solid state where the vinyl-acetate and vinyl-caprolactam moieties of this surfactant surround the drug while the PEG backbone forms the backing. This provides another potential mechanism for kinetic solubility enhancement.

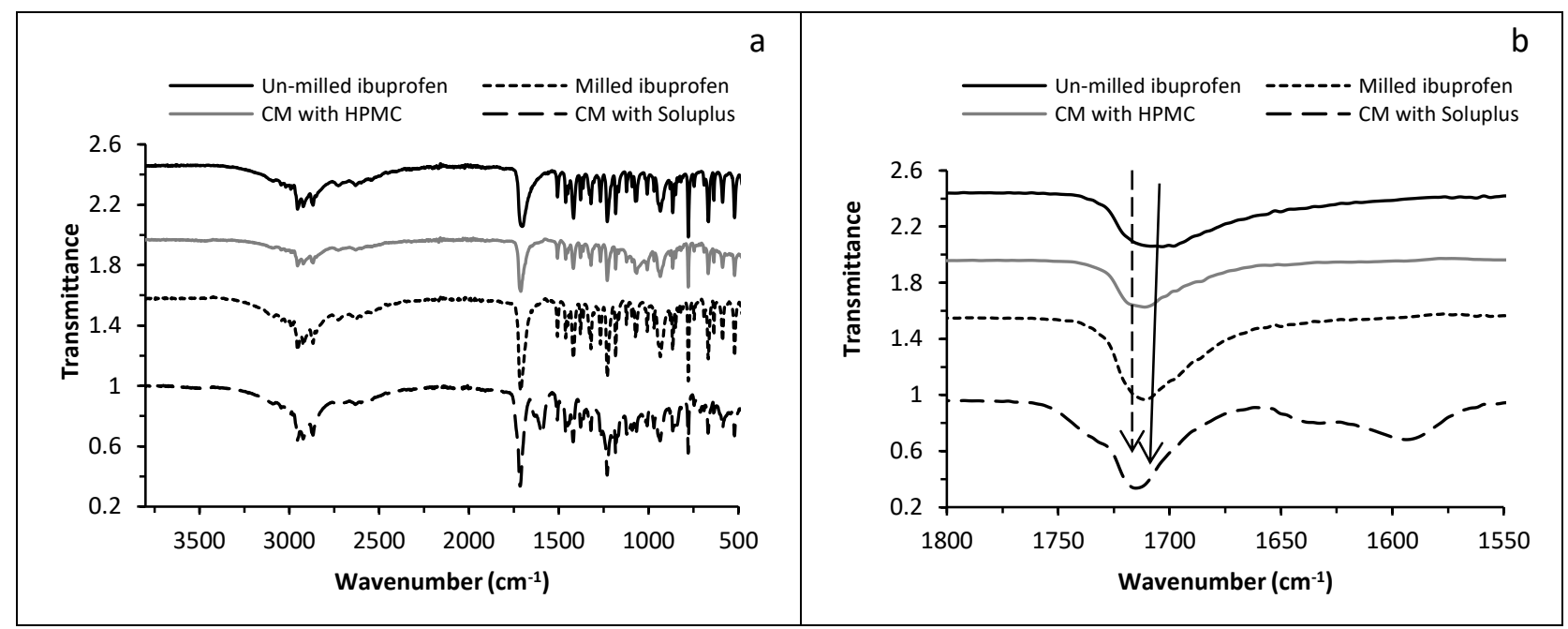

Fig. 7: a) IR spectra of un-milled, $15 \mathrm{~min}$ milled and its 1:1 co-milled mixtures with HPMC or Soluplus b) Carbonyl group peak from IR spectra of these samples, the shift in position of this peak is indicated by arrow heads.

\section{Conclusion}

This study indicates that co-milling with an excipient (either a polymer, i.e. HPMC, or surfactant, i.e. soluplus) effectively enhances the kinetic solubility and hence the dissolution rate of ibuprofen. This enhanced dissolution rate is considered to be the result of a number of interrelated phenomena that are likely to be working synergistically. Co-milling appears to enhance the reduction in particle size that is observed for ibuprofen alone but the differences between size reduction on co-milling with soluplus or HPMC don't appear to be significant and hence this mechanism is unlikely to explain the significantly increased dissolution rate one see for co-milled 
ibuprofen with soluplus. However, the \% decrease of the enthalpy of melting and the \% crystallinity (which is 55\% and 65\% for HPMC and Soluplus, respectively) will have a synergistic and therefore amplified effect on the dissolution rate through the alteration of the kinetic solubility. In addition the impact of soluplus on the FTIR spectrum of ibuprofen is further proof of the disruption to the ibuprofen structure in the solid state (producing a highly soluble solid state dispersion) provides further potential for increasing the kinetic solubility and dissolution rate, along with the enhanced solution state stability of ibuprofen in a soluplus rich solvent.

\section{Acknowledgement}

Amjad Hussain acknowledges the University of the Punjab, Lahore, Pakistan for PhD Scholarship.

\section{References}

Albadarin, A. B., Potter, C. B., Davis, M. T., Iqbal, J., Korde, S., Pagire, S., et al., 2017, Development of stability-enhanced ternary solid dispersions via combinations of hpmcp and soluplus ${ }^{\circledR}$ processed by hot melt extrusion Int. J. Pharm. 532, 603-611.

Barzegar-Jalali, M., Valizadeh, H., Shadbad, M.-R. S., Adibkia, K., Mohammadi, G., Farahani, A., et al., 2010, Co-grinding as an approach to enhance dissolution rate of a poorly water-soluble drug (gliclazide) Powdr. Tech. 197, 150-158.

Brittain, H. G., 2014. Thermodynamic vs. Kinetic solubility: Knowing which is which. American Pharmaceutical Review. Retrieved from

Brunton, L. L., Lazo, J. S., \& Parker, K. L. Goodman \& gilman's the pharmacological basis of therapeutics (Vol. 11): McGraw-Hill New York.2006

Caron, V., Hu, Y., Tajber, L., Erxleben, A., Corrigan, O. I., McArdle, P., et al., 2013, Amorphous solid dispersions of sulfonamide/soluplus ${ }^{\circledR}$ and sulfonamide/pvp prepared by ball milling AAPS PharmSciTech. 14, 464-474.

Chowdary, K., \& Susmitha, K., 2012, A factorial study on enhancement of solubility and dissolution rate of ibuprofen by $\beta$-cyclodextrin and solutol hs-15 Int. J. Res. Pharm. Chem. 2, 10431048.

Dudognon, E., Danède, F., Descamps, M., \& Correia, N. T., 2008, Evidence for a new crystalline phase of racemic ibuprofen Pharm. Res. 25, 2853-2858.

Erk, N., 2000, Quantitative analysis of chlorpheniramine maleate and phenylephrine hydrochloride in nasal drops by differential-derivative spectrophotometric, zero-crossing first derivative uv spectrophotometric and absorbance ratio methods J. Pharm. Biomed. Anal. 23, 1023-1031. 
Fisher, E. S. Milling of active pharmaceutical ingredients. In J. Swarbrick (Ed.), Encyclopedia of pharmaceutical technology (3rd ed.: Informa Healthcare, New York, USA.2007

Friedrich, H., Nada, A., \& Bodmeier, R., 2005, Solid state and dissolution rate characterization of co-ground mixtures of nifedipine and hydrophilic carriers Drug. Dev. Ind. Pharm. 31, 719-728.

Garg, A., Singh, S., Rao, V., Bindu, K., \& Balasubramaniam, J., 2009, Solid state interaction of raloxifene hcl with different hydrophilic carriers during co-grinding and its effect on dissolution rate Drug. Dev. Ind. Pharm. 35, 455-470.

Han, X., Ghoroi, C., To, D., Chen, Y., \& Davé, R., 2011, Simultaneous micronization and surface modification for improvement of flow and dissolution of drug particles Int. J. Pharm. 415, 185195.

Hasnain, M. S., \& Nayak, A. K., 2012, Solubility and dissolution enhancement of ibuprofen by solid dispersion technique using peg 6000-pvp k 30 combination carrier Bulg. J. Sci. Edu. 21, 118132.

Herbrink, M., Schellens, J. H. M., Beijnen, J. H., \& Nuijen, B., 2017, Improving the solubility of nilotinib through novel spray-dried solid dispersions Int. J. Pharm. 529, 294-302.

Jagadish, B., Yelchuri, R., Bindu, K., Tangi, H., Maroju, S., \& Rao, V. U., 2010, Enhanced dissolution and bioavailability of raloxifene hydrochloride by co-grinding with different superdisintegrants Chem. Pharm. Bull. 58, 293-300.

Ke, Z., Zhang, Z., Wu, H., Jia, X., \& Wang, Y., 2017, Optimization and evaluation of oridonin-loaded soluplus ${ }^{\circledR}$-pluronic p105 mixed micelles for oral administration Int. J. Pharm. 518, 193-202.

Kocbek, P., Baumgartner, S., \& Kristl, J., 2006, Preparation and evaluation of nanosuspensions for enhancing the dissolution of poorly soluble drugs Int. J. Pharm. 312, 179-186.

Krause, B., Villmow, T., Boldt, R., Mende, M., Petzold, G., \& Pötschke, P., 2011, Influence of dry grinding in a ball mill on the length of multiwalled carbon nanotubes and their dispersion and percolation behaviour in melt mixed polycarbonate composites Comp. Sci. Tech. 71, 1145-1153.

Larsson, I., \& Kristensen, H. G., 2000, Comminution of a brittle/ductile material in a micros ring mill Powdr. Tech. 107, 175-178.

Lin, S.-Y., Hsu, C.-H., \& Ke, W.-T., 2010, Solid-state transformation of different gabapentin polymorphs upon milling and co-milling Int. J. Pharm. 396, 83-90.

Madhuri Newa, K. H. B., Jong Oh Kim, Jong Seob IM, Jung Ae KIM,, \& Bong Kyu Yoo, J. S. W., Han Gon CHOI,* and Chul Soon Yong. 2008, Enhancement of solubility, dissolution and bioavailability of ibuprofen in solid dispersion systems Chem. Pharm. Bull. 56, 569-574.

Mallick, S., Pattnaik, S., Swain, K., De, P. K., Saha, A., Ghoshal, G., et al., 2008, Formation of physically stable amorphous phase of ibuprofen by solid state milling with kaolin Eur. J. Pharm. Biopharm. 68, 346-351. 
Mallick, S., Pattnaik, S., Swain, K., De, P. K., Saha, A., Mazumdar, P., et al., 2008, Physicochemical characterization of interaction of ibuprofen by solid-state milling with aluminum hydroxide Drug. Dev. Ind. Pharm. 34, 726-734.

Milhem, O. M., Myles, C., McKeown, N. B., Attwood, D., \& D'Emanuele, A., 2000, Polyamidoamine starburst ${ }^{\circledR}$ dendrimers as solubility enhancers Int. J. Pharm. 197, 239-241.

Mosharraf, M., \& Nyström, C., 1995, The effect of particle size and shape on the surface specific dissolution rate of microsized practically insoluble drugs Int. J. Pharm. 122, 35-47.

Nagy, Z. K., Balogh, A., Vajna, B., Farkas, A., Patyi, G., Kramarics, Á., et al., 2012, Comparison of electrospun and extruded soluplus ${ }^{\circledR}$-based solid dosage forms of improved dissolution J. Pharm. Sci. 101, 322-332.

Nallasivan, P. K., Saranya, K., Kumar, R. S., Jeevanantham, S., \& Venkatnarayanan, R., 2010, Simultaneous determination of ibuprofen and tizanidine in bulk drug and its combined dosage form by spectrophotometry Sch Res Lib. 2, 289-295.

Nandi, I., Bateson, M., Bari, M., \& Joshi, H., 2003, Synergistic effect of peg-400 and cyclodextrin to enhance solubility of progesterone AAPS PharmSciTech. 4, 1-5.

Newa, M., Bhandari, K., Oh, D., Kim, Y., Sung, J., Kim, J., et al., 2008, Enhanced dissolution of ibuprofen using solid dispersion with poloxamer-407 Arch. Pharm. Res. 31, 1497-1507.

Nokhodchi, A., Amire, O., \& Jelvehgari, M., 2010, Physico-mechanical and dissolution behaviours of ibuprofen crystals crystallized in the presence of various additives Daru. 18, 74.

Passerini, N., Albertini, B., González-Rodríguez, M. L., Cavallari, C., \& Rodriguez, L., 2002, Preparation and characterisation of ibuprofen-poloxamer 188 granules obtained by melt granulation European Journal of Pharmaceutical Sciences. 15, 71-78.

Patil, P. R., Rakesh, S. U., Dhabale, P. N., \& Burade, K. B., 2009, Simultaneous estimation of ramipril and amlodipine by uv spectrophotometric method Res. J. Pharm. Tech. 2, 304-307.

Plakkot, S., de Matas, M., York, P., Saunders, M., \& Sulaiman, B., 2011, Comminution of ibuprofen to produce nano-particles for rapid dissolution Int. J. Pharm. 415, 307-314.

Pokharkar, V. B., Mandpe, L. P., Padamwar, M. N., Ambike, A. A., Mahadik, K. R., \& Paradkar, A., 2006, Development, characterization and stabilization of amorphous form of a low tg drug Powdr. Tech. 167, 20-25.

Qiao, N., Wang, K., Schlindwein, W., Davies, A., \& Li, M., 2013, In-situ monitoring of carbamazepine-nicotinamide co-crystal intrinsic dissolution behaviour Eur. J. Pharm. Biopharm. $83,415-426$.

Saleh, W., Denis, D., Jean-Louis, D., Gilles, B., \& Bernard, B., 2008, The improvement of ibuprofen dissolution rate through microparticles spray drying processed in an aqueous system Drug. Dev. Ind. Pharm. 34, 485-491. 
Salústio, P. J., Cabral-Marques, H. M., Costa, P. C., \& Pinto, J. F., 2011, Comparison of ibuprofen release from minitablets and capsules containing ibuprofen: B-cyclodextrin complex Eur. J. Pharm. Biopharm. 78, 58-66.

Smith, G., Hussain, A., Bukhari, N., \& Ermolina, I., 2015, Quantification of residual crystallinity of ball-milled, commercially available, anhydrous $\beta$-lactose by differential scanning calorimetry and terahertz spectroscopy J. Therm. Anal. Calorm., 1-7.

Szafraniec, J., Antosik, A., Knapik-Kowalczuk, J., Kurek, M., Syrek, K., Chmiel, K., et al., 2017, Planetary ball milling and supercritical fluid technology as a way to enhance dissolution of bicalutamide Int. J. Pharm. 533, 470-479.

Vogt, M., Kunath, K., \& Dressman, J. B., 2008, Dissolution improvement of four poorly water soluble drugs by co-grinding with commonly used excipients Eur. J. Pharm. Biopharm. 68, 330337.

Vogt, M., Vertzoni, M., Kunath, K., Reppas, C., \& Dressman, J. B., 2008, Co-grinding enhances the oral bioavailability of emd-57033, a poorly water soluble drug in dogs Eur. J. Pharm. Biopharm. 68, 338-345.

Williams, A. C., Timmins, P., Lu, M., \& Forbes, R. T., 2005, Disorder and dissolution enhancement: Deposition of ibuprofen on to insoluble polymers European Journal of Pharmaceutical Sciences. 26, 288-294. 
Appendix 1

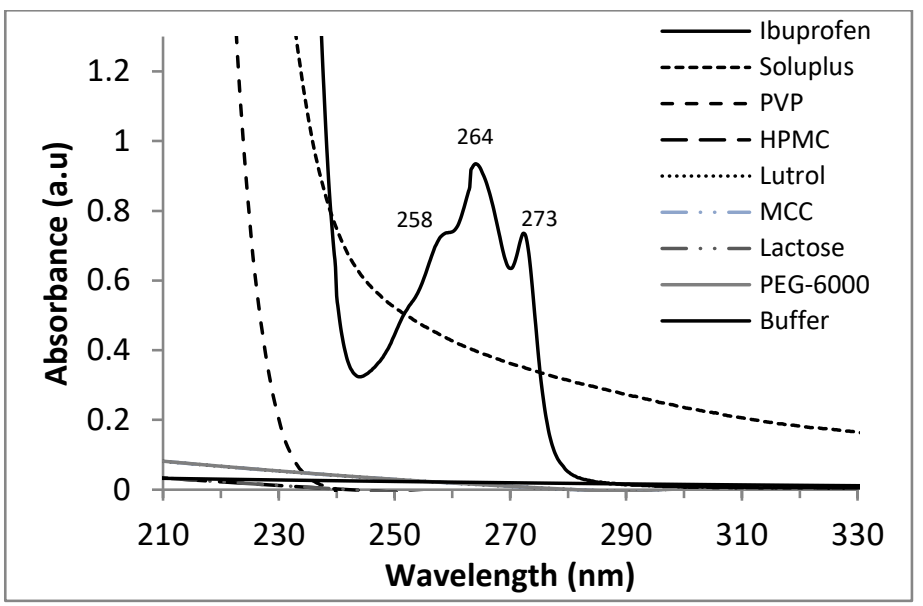

Appendix 1: Overlaid UV spectra of $0.05 \% \mathrm{w} / \mathrm{v}$ solutions of ibuprofen (showing two peaks at $273 \& 264 \mathrm{~nm}$ and a shoulder at $258 \mathrm{~nm}$ ) and different excipients in phosphate buffer (pH 7.4). 
Appendix 2

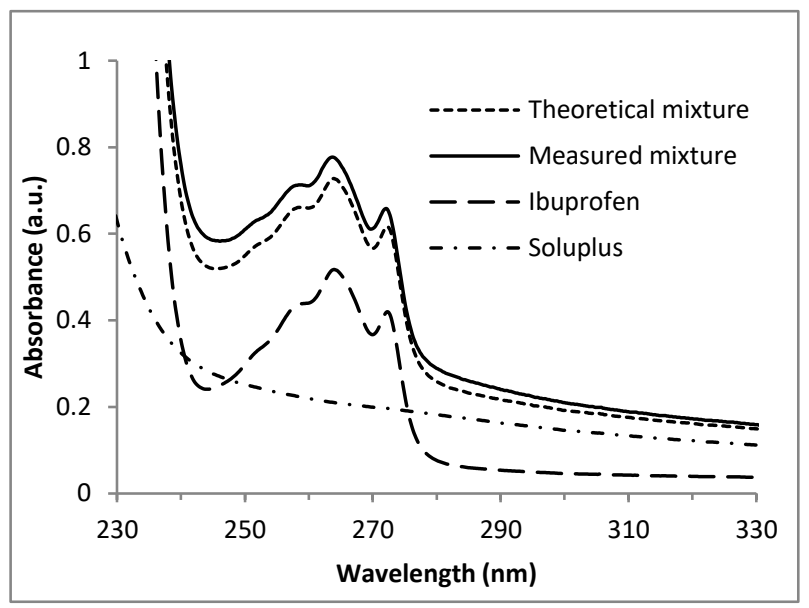

Appendix 2: UV spectra of $\mathbf{0 . 0 2 5 \%}$ Ibuprofen solution (dash line); $\mathbf{0 . 0 1 2 5 \%}$ Soluplus solution (dash-dot line); mixture of solid in liquid solution including $\mathbf{0 . 0 2 5 \%}$ Ibuprofen and $\mathbf{0 . 0 1 2 5 \%}$ Soluplus (1:1) (dot line); and theoretical spectrum of mixture of Soluplus and Ibuprofen estimated as a sum of individual spectra of $\mathbf{0 . 0 2 5 \%}$ Ibuprofen and $\mathbf{0 . 0 1 2 5 \%}$ Soluplus (solid line). 


\section{Appendix 3}

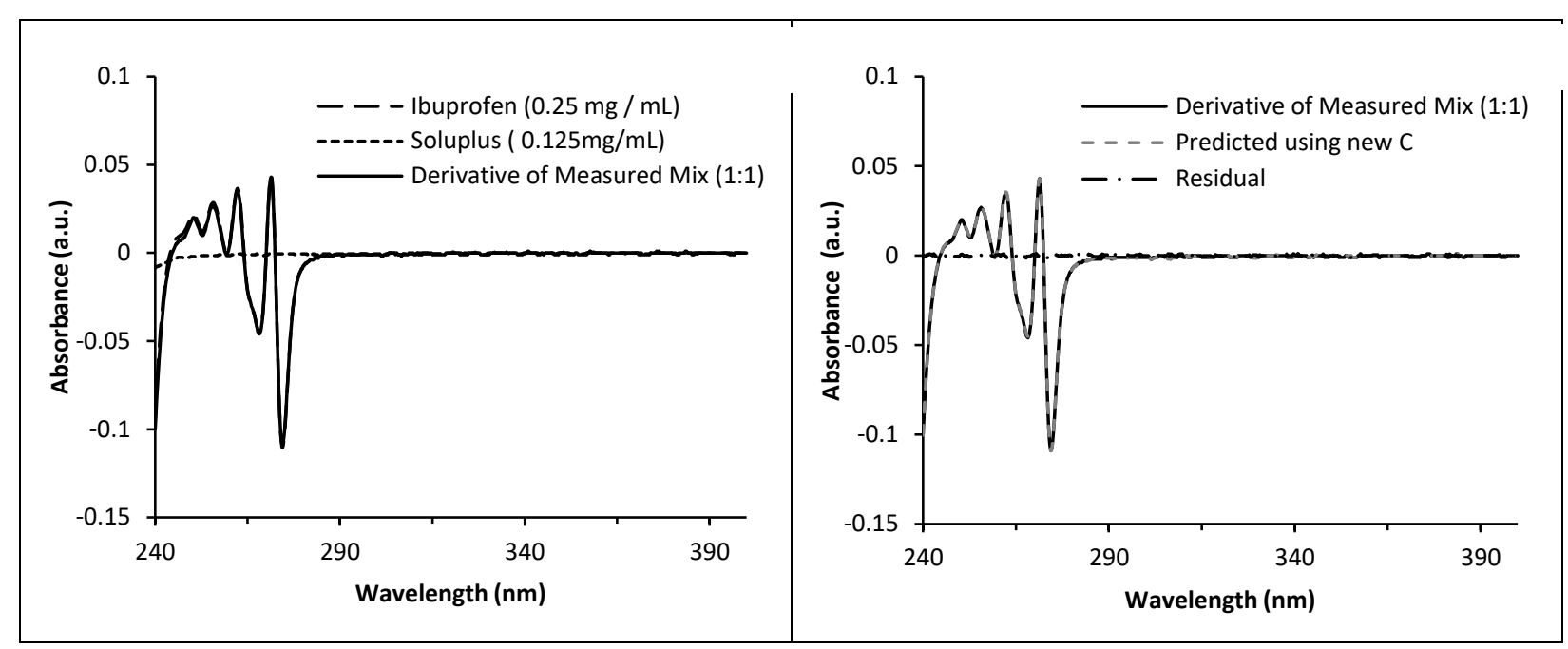

Appendix 3: Reference derivative spectra of ibuprofen and soluplus and their 1:1 mixture (a), predicted derivative spectrum by multivariate analysis overlaid by the spectrum of mixture (b) Both spectra have been truncated between $240-400 \mathrm{~nm}$. 